\title{
Planetarium commute accessibility in the United States of America
}

\author{
Alexander A. Kaurov ${ }^{1}$, Vyacheslav Bazhenov ${ }^{2}$, and Mark SubbaRao ${ }^{3}$ \\ ${ }^{1}$ Institute for Advanced Study \\ ${ }^{2}$ Affiliation not available \\ ${ }^{3}$ International Planetarium Society
}

May 20, 2021

\begin{abstract}
The COVID-19 global pandemic unprecedently disturbed the education system in the United States and lead to the closure of all planetariums that were providing immersive science communication. This situation motivates us to examine how accessible the planetarium facilities were before the pandemic. We investigate the most important socioeconomic and geographical factors that affect the planetarium accessibility using the U.S. Census Bureau data and the commute time to the nearest planetarium for each ZIP Code Tabulated Area. We show the magnitude of the effect of permanent closure of a fraction of planetariums. Our study can be informative for strategizing the pandemic response.
\end{abstract}

\section{Introduction}

Traditional (opto-mechanical) planetariums are immersive facilities designed to simulate the night sky. Modern digital planetarium technology has expanded upon those capabilities, adding the ability to display a wide range of scientific phenomena. These facilities have become important tools for a wide range of scientific communication, helping to keep the public engaged on the latest scientific progress. Museums are some of the most trusted sources of scientific information. The National Awareness, Attitude and Usage Study of Visitor-Serving Organizations (The National Awareness, Attitude and Usage Study of Visitor-Serving Organizations (NAAU), n.d.) shows that museums and science centers are a significantly more trusted source of scientific information than either the government or media. Furthermore, there are indications that in the COVID-19 global pandemic is widening this divide(Dilenschneider, 2020).

The United States has the more planetariums than any other country in the world. That network is coordinated of seven regional planetarium societies, all of whom are affiliates of the International Planetarium Society. This network of planetariums has the opportunity to operate in coordinated fashion(Subbarao, 2020), to keep the public informed on scientific issues that need informed input as part of the public debate, such as climate change or the impact of satellite mega-constellations. In the planetarium information is frequently presented by a live presenter. The presence of this presenter allows for a dialog between the presenter and the public and the presenter, something that is not possible in one-way media. Two-way communication allows the presenter to shift the presentation to reflect the audience's needs(Yuan et al., 2017). This ability to tailor the presentation is especially important when presenting potentially controversial topics, or to audiences who are likely to be skeptical of science.

Given the important role that planetariums can play in keeping the public informed of current science this study aims to determine how accessible are planetariums to the US population. The current COVID-19 
pandemic has had a devastating effect on the financial health of planetariums across the world(Subbarao, 2020), with essentially all planetariums needing to close for an extended basis. We also simulate how the loss of planetariums due to the COVID-19 pandemic would impact planetarium accessibility across the US.

In sections 2 we describe the methods and their limitations. In section 3 we show the results of our analysis of the planetarium commute accessibility with a fraction of planetariums closed averaged over the United States, then we split the data into four geographical regions, and finally we split the population geographically into low and high income levels. In section 4 we present our conclusions.

\section{Methods and Limitations}

To estimate the commute accessibility, we combine the following datasets in our study.

\section{Planetariums}

We adopt the catalog of planetariums by Association des Planétariums de Langue Française (APLF)(Worldwide Planetariums Database, n.d.). It contains the locations as well as dome diameters and seat count for the planetariums worldwide. The planetarium database is maintained by APLF, and there is no public documentation on the methods used to assemble it. Our manual check shows that the catalog is representative, and all major planetariums are listed properly. For the planetariums with multiple domes we consider only the largest one. In total, there are 737 facilities among which 232 have 'school' or 'schools' in their name, 122 have 'college' and 151 have 'university', and the rest 220 are associated with museums and dedicated planetariums.

To estimate the effect of planetarium closures we divide the planetariums in half by the median dome size. This cut happens to be at 9.1 meters and there are many planetariums that have exactly 9.1-meter dome, so we randomly distribute them between two groups. The motivation is to separate large facilities that more often have extensive programming and are more financially robust, and the smaller ones that are often located in schools and on college campuses and not necessarily provide public programs, especially after the pandemic. 


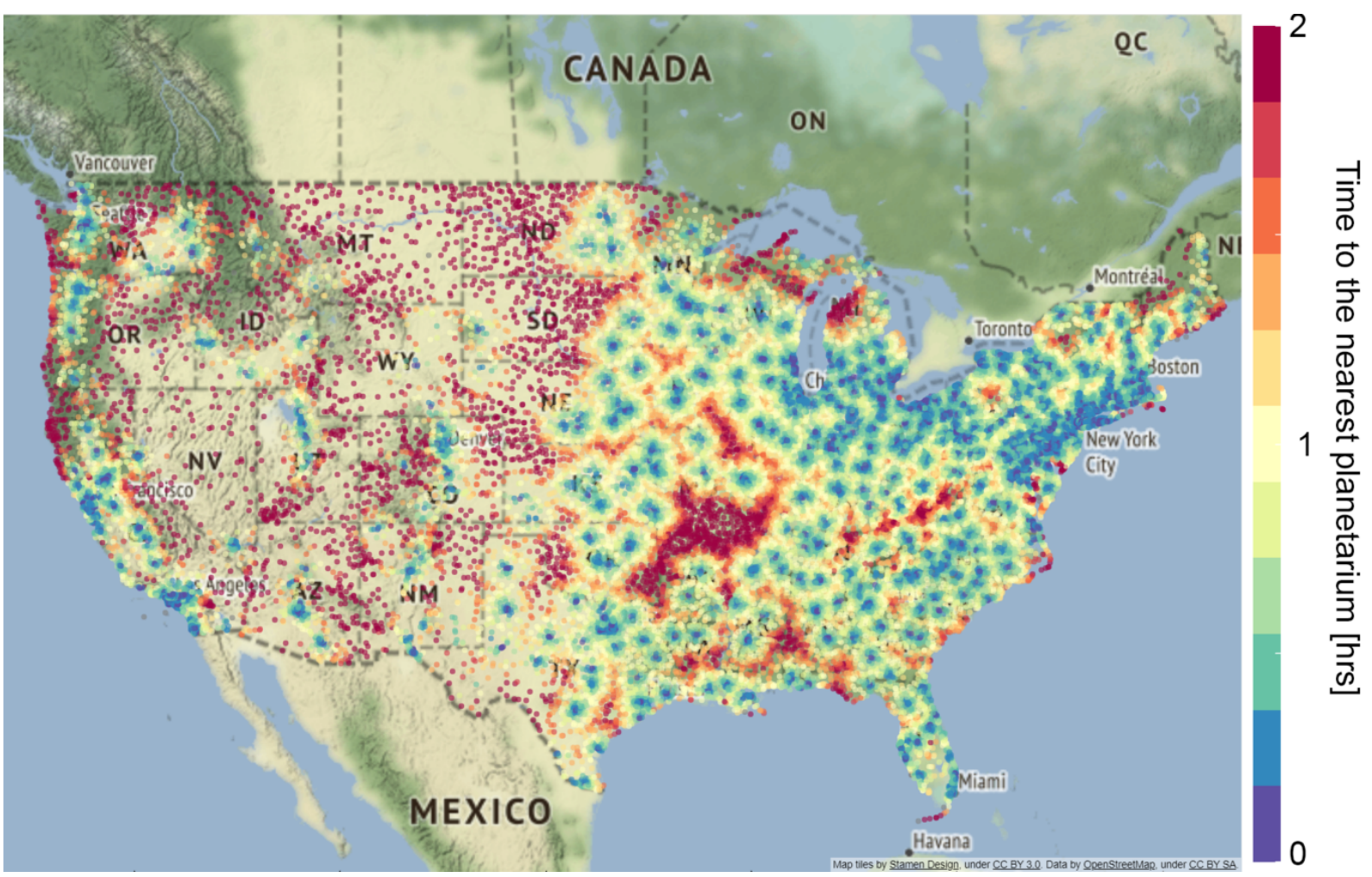

Figure 1: Commute time from the center of each ZIP code to the nearest planetarium by car. Google Maps API is used to estimate time and we assume the most optimistic scenario with no traffic. All times above 2 hours are marked with the same color. Red squares represent the planetariums from APLF catalog.

\section{Census data}

Census data is adopted from the American Community Survey (ACS) 5-years estimate (2014-2018)(Bureau, 2018) and retrieved with censusdata Python package(Python package 'censusdata', n.d.). For this study, we use the total population, information on race and Hispanic origin, and household income in the past 12 months (in 2018 inflation-adjusted dollars) in ZIP Code Tabulation Areas (ZCTAs).

Differences in ZCTA's sizes and methods of their allocations between the states, which is evident from Figure 1 , may cause biases in the summary statistics. We do not have access to the population distribution on the level finer than ZCTA.

\section{Traffic}

The routing estimates are taken from Google Maps API(Google Maps API, n.d.). We evaluate the commute time from ZCTA's geographical center to the nearest planetarium. When the center happens to be in the middle of unpopulated area (with no roads) or in the water, Google Maps' car routing algorithm automatically finds the nearest road and calculates driving time from there.

We are strictly limited by the number of Google Maps API requests that are not free. It prevents us from doing geographical sampling; and therefore, by taking the center of the ZCTAs we effectively neglect any inhomogeneity on scales smaller than ZCTA. Also, we cannot do temporal averaging and therefore we adopted local 9 AM on 08/05/2020 (Wednesday). Any temporary road closures or other traffic conditions may affect local estimates. Google Maps API does not allow one to access archival traffic information so we 
cannot assess the traffic before the pandemic; thus, we assume the most optimistic scenario with no traffic because current traffic is not representative.

We do not study public transportation which is unavailable in many regions of the United States and differs significantly from city to city.

In Figure 1 we show the distribution of planetariums and commute times from each ZCTA to the nearest planetarium.

\section{Results}

\section{US-wide summary}

We calculate summary statistics for the United States including District of Columbia and Puerto Rico. In Figure 2 we show the cumulative distribution of the commute time by car with no traffic. It shows that $89 \%$ of 326 million population has a planetarium within 60-minute driving distance and this number falls to $81 \%$ if half of the planetariums is closed.

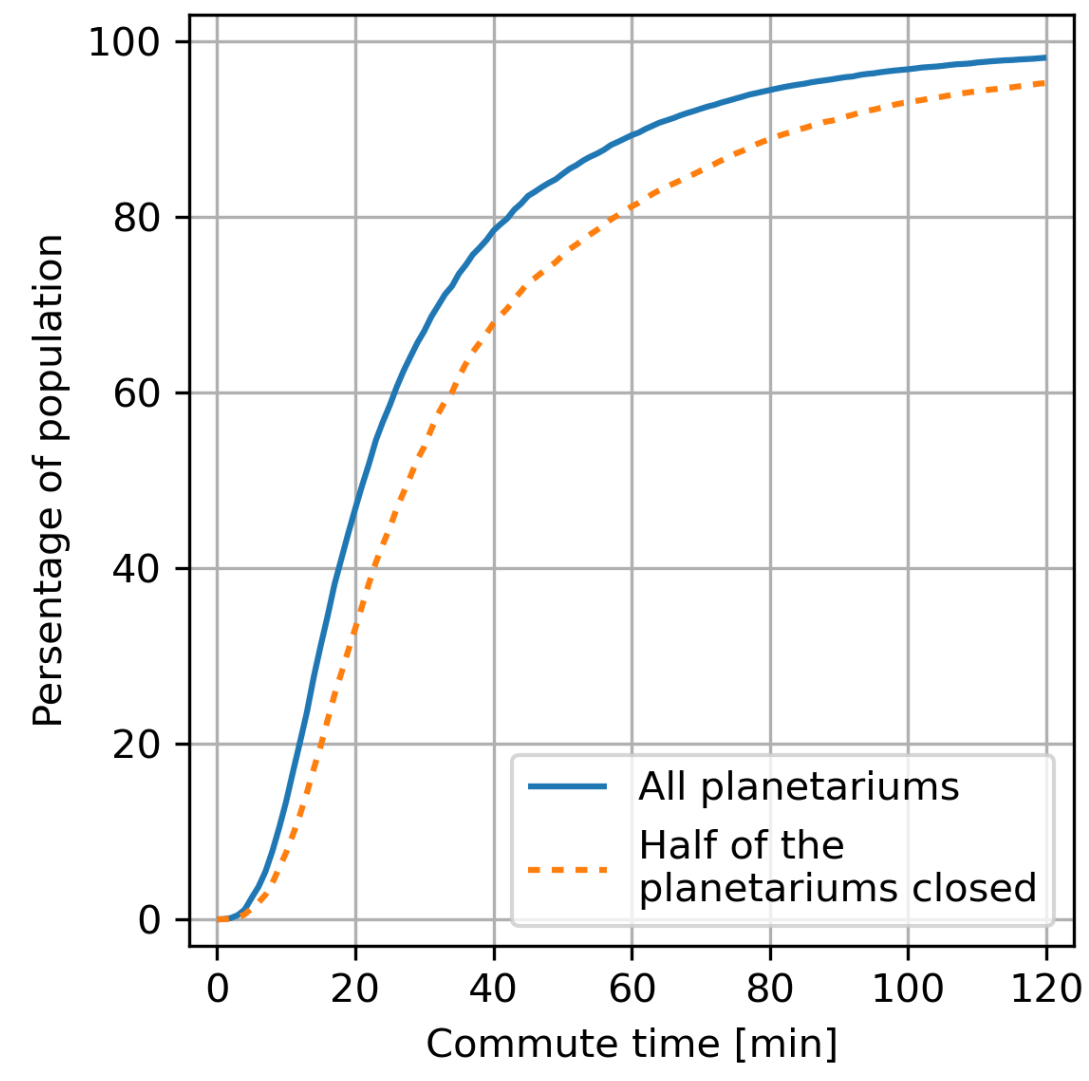

Figure 2: Commute accessibility of the planetariums in the United States by car with no traffic. The dashed line shows the effect of $50 \%$ of smaller planetariums closure. 


\section{Geographical regions}

The map in Figure 1 shows that there are large regions that are poorly populated by the planetariums. Those regions are associated with low population density. To highlight the geographical differences, we consider four geographical regions, and we show the results in Figure 3. The left panel shows that the Northeast region has significantly better accessibility and it is apparent from the density of the planetariums' distribution in Figure 1. The right panel shows that after we close $50 \%$ of smaller planetariums, the advantage of the Northeast region is diminished, meaning that many of the planetariums in that area are small.
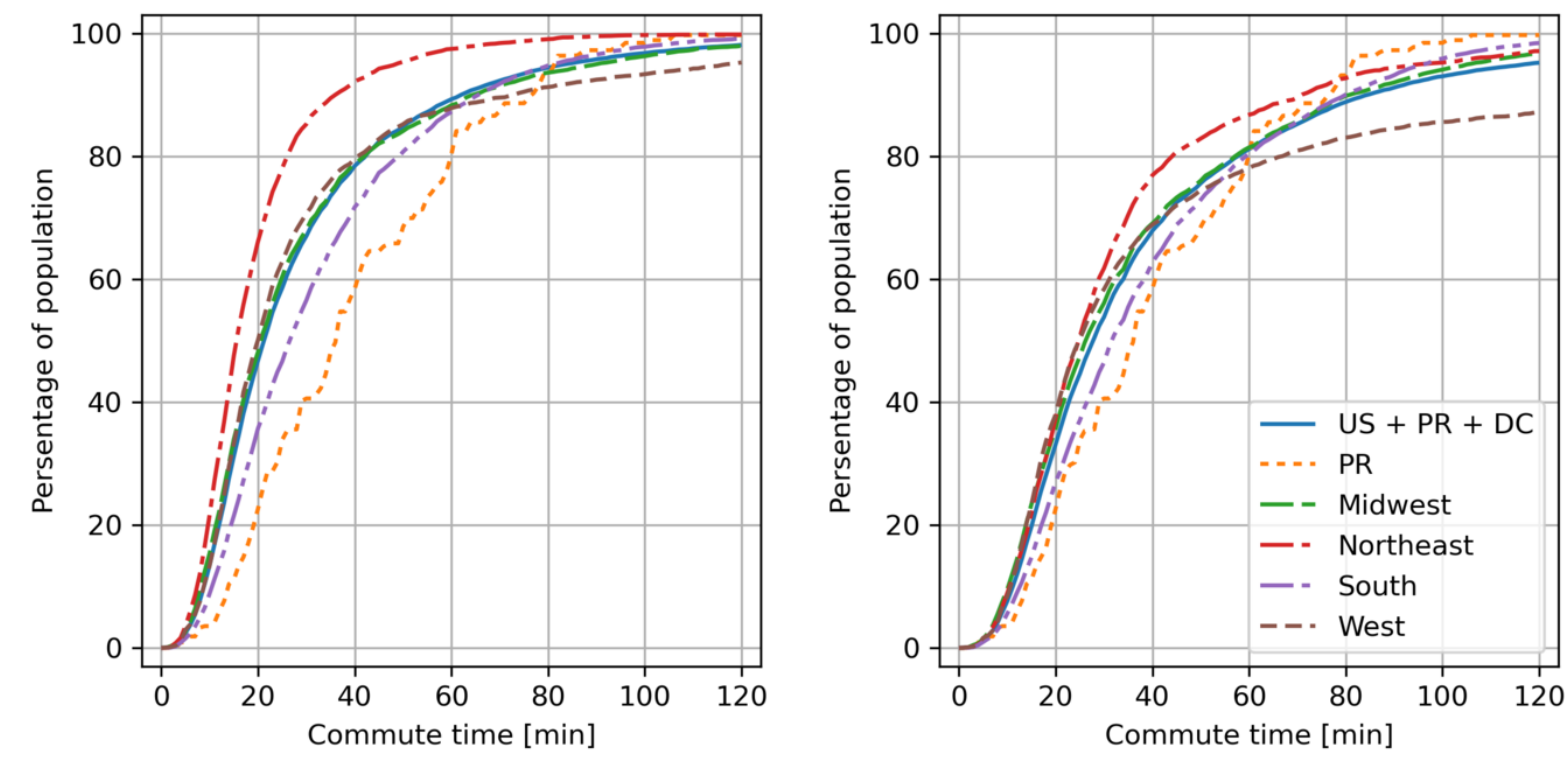

Figure 3: Commute accessibility of the planetariums in the United States by car with no traffic in four geographical regions and Puerto Rico. The second panel shows the effect of $50 \%$ of smaller planetariums closure.

In Figure 4 we show the United States colored by the percentage of population that does not have a planetarium in a close, 30-minute drive, proximity. 

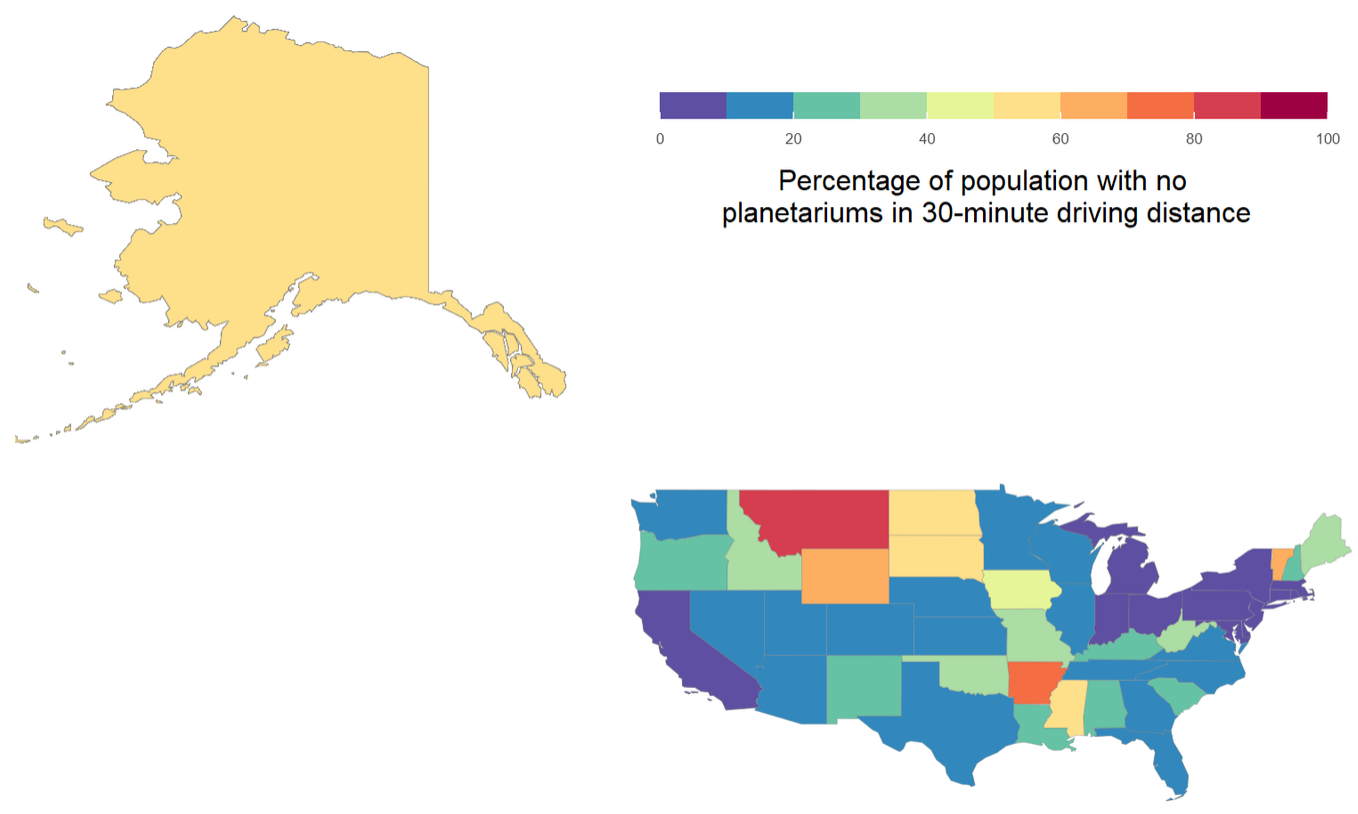

Figure 4: The percentage of population that have no planetariums in 30 min driving distance in assumption of no traffic and all the planetariums in our catalog open.

\section{Household Income}

In this sub-section we consider 120,9 million households instead of individual people since the income data is reported per household. We divide them into 3 groups: below $\$ 40,000$ per year (34\% of households), between $\$ 40,000$ and $\$ 100,000(38 \%)$, and above $\$ 100,000(28 \%)$. The result is shown in Figure 5 and highlights the effect of the low-income on the accessibility of the planetarium facilities. 

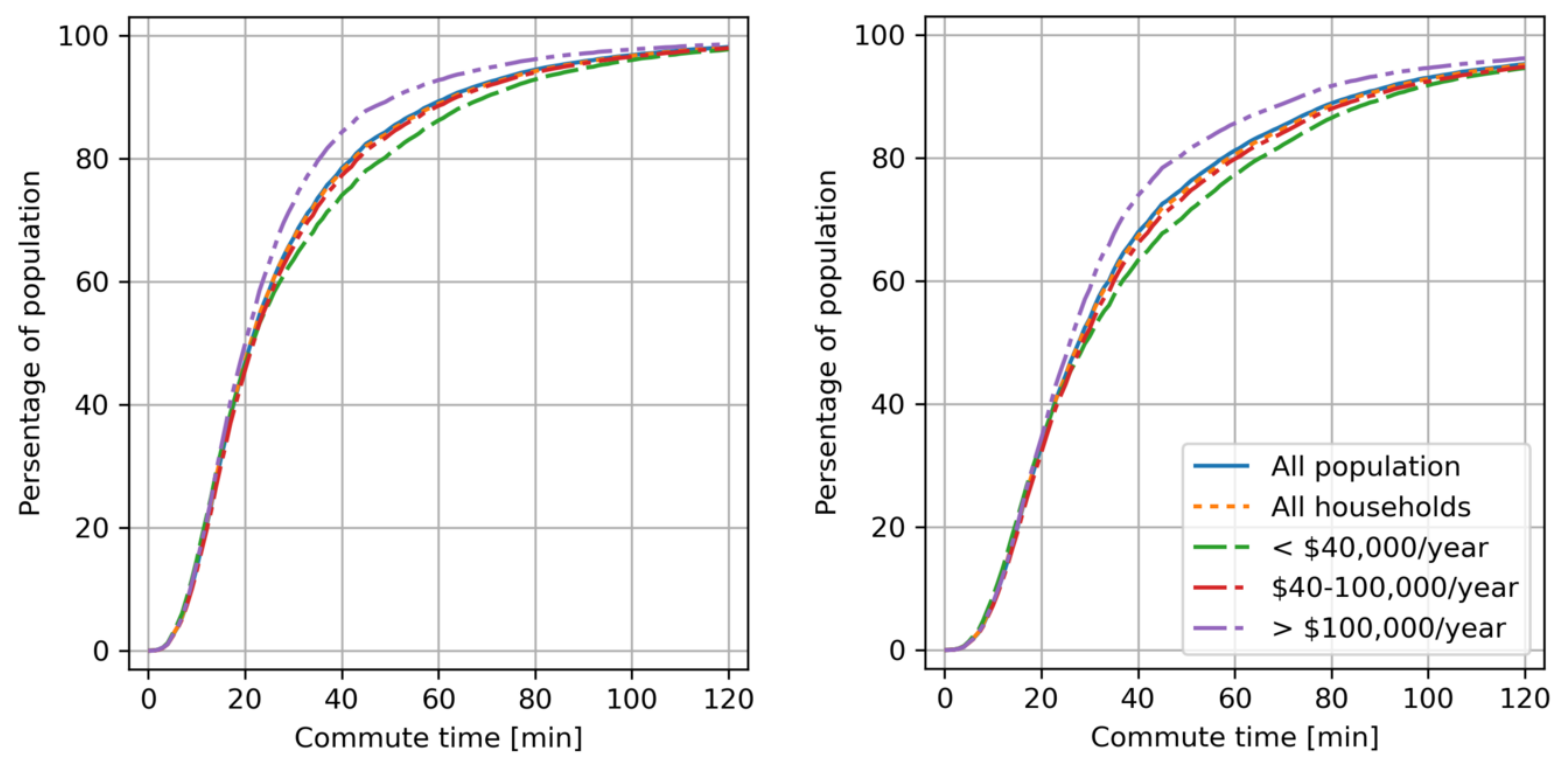

Figure 5: Commute time by car with no traffic to the nearest planetarium for the households with different income. The second panel shows the effect of $50 \%$ of smaller planetariums closure.

\section{Race and Hispanic origin}

The population is divided into the following races by the Census Bureau: White (72.7\%), Black or African American (12.7\%), American Indian and Alaska Native (0.83\%), Asian (5.4\%), Native Hawaiian and Other Pacific Islander $(0.18 \%)$, some other race $(5.0 \%)$, two or more races $(3.3 \%)$. The main driving force of the trends in Figure 6 is the geographical distribution. The American Indian and Alaska Native live further away from urban areas, and therefore have poorest access. The white race is the most widespread and distributed across all states and suburban areas; therefore, their distribution is very close to the mean but slightly lower. 

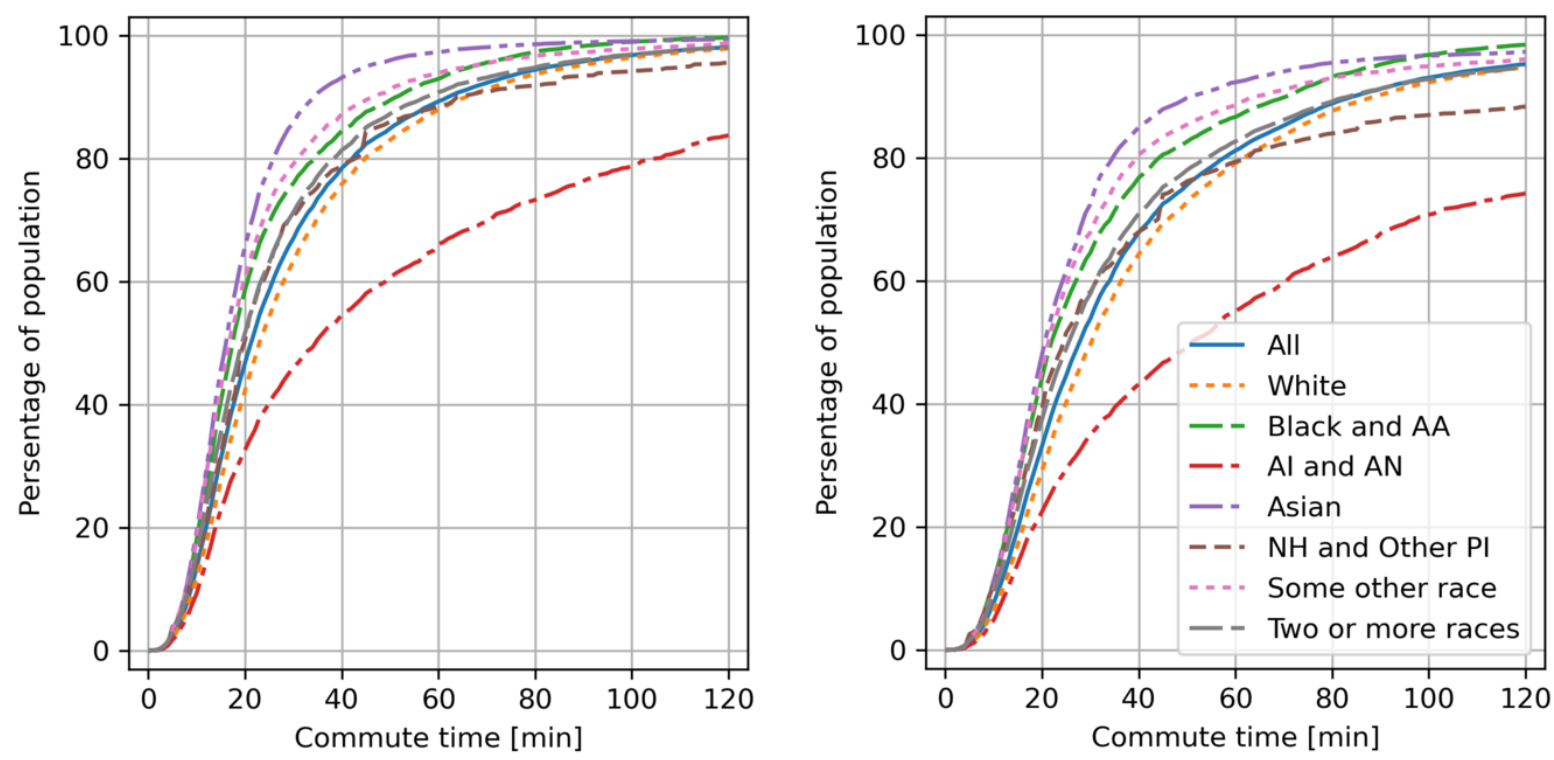

Figure 6: The commute access for different races: White, Black or African American (Black and AA), American Indian and Alaska Native (AI and AN), Asian, Native Hawaiian and Other Pacific Islander (NH and Other PI), some other race, and two or more races. The second panel shows the effect of $50 \%$ of smaller planetariums closure.

$18.7 \%$ of population identify themselves as Hispanic, Latino or Spanish and may be any race. In Figure 7 we show their access to the planetariums that is slightly better compared to Non-Hispanic, and this difference increases when the half of the planetariums is closed.

In 2014 blacks or African Americans accounted for $13 \%$ of the US population but only $5 \%$ of the degrees earned in the physical sciences. Hispanics accounted for 17\% of the US population but earned only $8 \%$ of the physical sciences degrees(The National Center for Science and Engineering Statistics (NCSES), n.d.). These groups that are underrepresented in STEM actually have better than average access to planetariums. This high level of accessibility shows that planetariums are well positioned to enhance the participation in STEM fields among blacks and African Americans, and Hispanics. 

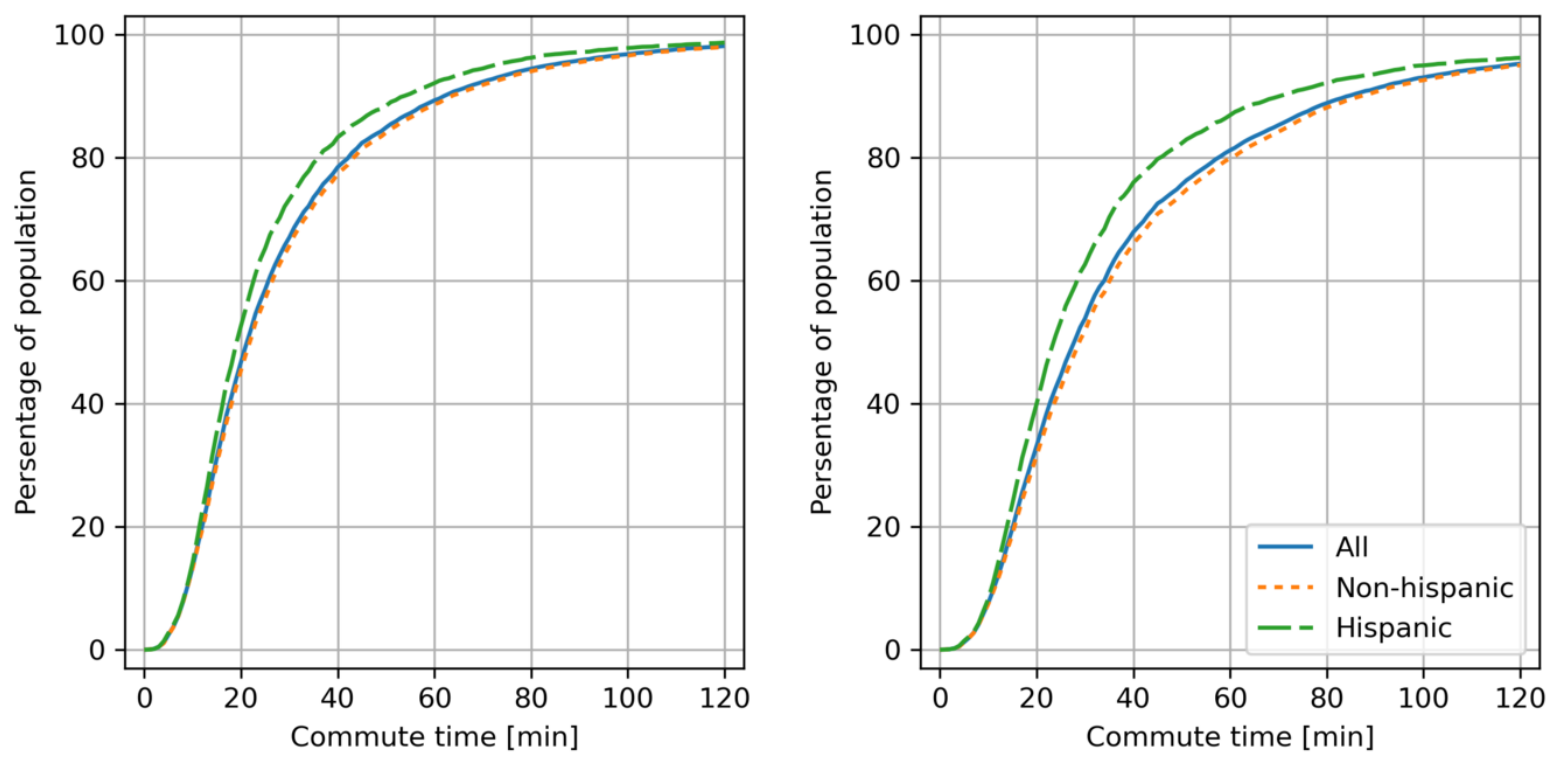

Figure 7: The commute access for Hispanic, Latino or Spanish and Non-Hispanic population. The second panel shows the effect of $50 \%$ of smaller planetariums closure.

\section{Conclusions}

The USA has a strong network of planetariums and most of the population is within an easy drive of a planetarium visit. Wealthier people have significantly better access than poorer groups. Access varies among racial and ethnic groups. Blacks and African Americans, and Hispanics, both have better average access to planetariums. Planetariums are currently struggling due to the COVID-19 pandemic. Closure of half of the planetariums would significantly disrupt this planetarium network and increase the fraction of the population to whom planetariums are inaccessible.

We summarize our findings in Table 1 below and in the following bullet points:

- $67 \%(89 \%)$ of population lives within 30 (60) minutes driving distance from a planetarium, leaving $11 \%$ that do not have easy access to the planetariums. Among those people are those who have no road access at all. Closure of smaller $50 \%$ of planetariums leads to these numbers $54 \%$ (81\%).

- Separation into the geographical regions shows that Northeast region has better accessibility to the planetariums with $85 \%(97 \%)$ and this advantage is mostly driven by the abundance of small planetariums.

- The households with income below $\$ 40,000$ per year that account for $34 \%$ of households $64 \%$ ( $86 \%$ ) accessibility. Those with income above $\$ 100,000$ that account for $28 \%$ of households have marginally better access with $67 \%(89 \%)$.

- The race group with the poorest access to the planetariums is "American Indian and Alaska Native". For them only $46 \%(66 \%)$ have a planetarium within $30(60)$ minutes driving distance. The Asian race group has the best access with $86 \%$ (97\%).

- People of Hispanic, Latino and Spanish origin have a better access with $73 \%$ (92\%) compared to Non-Hispanic with $66 \%(89 \%)$. 


\begin{tabular}{|c|c|c|c|c|c|c|c|}
\hline \multirow{2}{*}{\multicolumn{2}{|c|}{ Selection }} & \multicolumn{3}{|c|}{ All planetariums } & \multicolumn{3}{|c|}{$50 \%$ of smaller planetariums closed } \\
\hline & & \multirow{3}{*}{$\begin{array}{l}\text { within } \\
30 \text { min } \\
67\end{array}$} & \multirow{3}{*}{$\begin{array}{l}\text { within } \\
60 \text { min } \\
89\end{array}$} & \multirow{3}{*}{$\begin{array}{l}\text { more than } \\
60 \text { min } \\
11\end{array}$} & \multirow{3}{*}{$\begin{array}{l}\text { within } \\
30 \text { min } \\
54\end{array}$} & \multirow{3}{*}{$\begin{array}{l}\text { within } \\
60 \text { min } \\
81\end{array}$} & \multirow{3}{*}{$\begin{array}{l}\text { more than } \\
60 \text { min } \\
19\end{array}$} \\
\hline & & & & & & & \\
\hline \multirow{6}{*}{$\begin{array}{l}\text { USA (all pop } \\
\text { Geographical } \\
\text { region }\end{array}$} & tion) & & & & & & \\
\hline & Northeast & 85 & 97 & 3 & 62 & 87 & 13 \\
\hline & West & 71 & 88 & 12 & 58 & 78 & 22 \\
\hline & Midwest & 68 & 88 & 12 & 56 & 81 & 19 \\
\hline & South & 56 & 87 & 13 & 46 & 80 & 20 \\
\hline & Puerto Rico & 41 & 80 & 20 & 41 & 80 & 20 \\
\hline \multirow{4}{*}{ Income } & All households & 67 & 89 & 11 & 54 & 81 & 19 \\
\hline & $<\$ 40,000 /$ year & 64 & 86 & 14 & 51 & 77 & 23 \\
\hline & $\$ 40-100,000 /$ year & 66 & 89 & 11 & 52 & 80 & 20 \\
\hline & $>\$ 100,000 /$ year & 73 & 93 & 7 & 59 & 86 & 14 \\
\hline \multirow{7}{*}{ Race } & White & 63 & 88 & 12 & 50 & 79 & 21 \\
\hline & $\begin{array}{l}\text { Black or } \\
\text { African American }\end{array}$ & 76 & 93 & 7 & 65 & 87 & 13 \\
\hline & $\begin{array}{l}\text { American Indian and } \\
\text { Alaska Native }\end{array}$ & 46 & 66 & 34 & 35 & 55 & 45 \\
\hline & Asian & 86 & 97 & 3 & 72 & 92 & 8 \\
\hline & $\begin{array}{l}\text { Native Hawaiian and } \\
\text { Other Pacific Islander }\end{array}$ & 71 & 88 & 12 & 58 & 79 & 21 \\
\hline & some other race & 79 & 94 & 6 & 68 & 89 & 11 \\
\hline & two or more races & 71 & 91 & 9 & 58 & 83 & 17 \\
\hline \multirow[t]{2}{*}{$\begin{array}{l}\text { Hispanic } \\
\text { origin }\end{array}$} & $\begin{array}{l}\text { Hispanic, } \\
\text { Latino or Spanish }\end{array}$ & 73 & 92 & 8 & 63 & 87 & 13 \\
\hline & Non-Hispanic & 66 & 89 & 11 & 52 & 80 & 20 \\
\hline
\end{tabular}

Table 1: The summary of planetarium commute accessibility in the United States of America in percents. All calculations assume commute by car with no traffic.

Further studies may include search of the 'planetarium deserts', and the determination of the optimal placement for new planetariums.

More detailed information for the individual states and cities can be generated upon reasonable request. We are open for the collaborations to extend this study to other countries. 


\section{Acknowledgements}

AAK is supported by the IBM Einstein fellowship.

\section{References}

https://www . impacts-iq. com/naau

Data Shows Increased Credibility of Museums During the Pandemic. (2020). https://www.colleendilen . com/2020/07/08/data-shows-increased-credibility-of-museums-during-the-pandemic-data/

Effectively Coordinating Museums and Planetariums Worldwide. (2020). IAU General Assembly, 536-541. https://doi.org/10.1017/S1743921319005301

Two-way communication between scientists and the public: a view from science communication trainers in North America. (2017). International Journal of Science Education Part B, 7(4), 341-355. https: //doi.org/10.1080/21548455.2017.1350789

COVID-19: How are Planetariums Affected. (2020). Planetarian, 49(2). https://www.ips-planetarium. org/resource/resmgr/planetarian/202006planetarian.pdf

http://www. aplf-planetariums.org/en/index.php

2014-2018 American Community Survey 5-year estimates. (2018).

https://github.com/jtleider/censusdata

https://developers.google.com/maps/documentation

Women, Minorities, and Persons with Disabilities in Science and Engineering. https://www.nsf.gov/ statistics/2017/nsf17310/digest/introduction/ 